\title{
Long-Term Effects of Combined Submucous Turbinectomy and Posterior Nasal Neurectomy in Patients with Allergic Rhinitis
}

\author{
Kazuhiro Ogi ${ }^{1,2}$ - Yasuhiro Manabe ${ }^{1} \cdot$ Shigehito Mori $^{3} \cdot$ Yukihiro Kimura $^{2} \cdot$ Takahiro Tokunaga $^{1,2} \cdot$ Yukinori Kato $^{2}$. \\ Tetsuji Takabayashi ${ }^{2} \cdot$ Norihiko Narita $^{2} \cdot$ Shigeharu Fujieda ${ }^{2}$
}

Accepted: 29 May 2019 / Published online: 8 June 2019

(C) The Author(s) 2019

\begin{abstract}
Submucous inferior turbinectomy (ST) can be combined with posterior nasal neurectomy (PNN) to treat intractable allergic rhinitis (AR) and vasomotor rhinitis (VR). This study assessed the long-term effects and complications of this surgical intervention. We enrolled 127 patients who had undergone combined ST and PNN. We mailed these patients card questionnaires. Of these questionnaires, we excluded the 5 patients with VR and 26 patients who had undergone concomitant endoscopic sinus surgery (ESS), because they had both AR or VR and sinusitis. Then, we assessed the questionnaire score of 31 patients with AR who had undergone combined ST and PNN. Postoperative bleeding was investigated in 127 subjects by medical records. Moreover, we compared the incidence of numbness in the palate, cheeks, or teeth between patients who had undergone PNN and those who had not. We found that $77.4 \%$ of the AR patients were satisfied with the improvement of their nasal symptoms after the operation. Although seven patients had been receiving medical treatment from an otolaryngology clinic every month before the operation, none needed any such treatment after the operation. One year postoperatively, all symptom scores were statistically decreased. However, the scores increased over time, although there was a significant improvement in nasal obstruction more than 3 years after the operation $(P<0.01)$. Five patients $(3.9 \%)$ among 127 patients who had undergone PNN were treated for postoperative bleeding. Patients who had undergone PNN did not differ significantly from those who had not in terms of postoperative numbness in the palate, cheeks, or teeth. Endoscopic ST combined with PNN is an effective treatment for intractable AR. Further studies are required to elucidate the efficacy of this intervention.
\end{abstract}

Keywords Surgical outcome $\cdot$ Submucous inferior turbinectomy $\cdot$ Posterior nasal neurectomy $\cdot$ Allergic rhinitis

This article is part of the Topical Collection on Surgery

\author{
Kazuhiro Ogi \\ ogikazu@shinseikai.or.jp; ogikazu@u-fukui.ac.jp \\ Yasuhiro Manabe \\ manabe@shinseikai.or.jp \\ Shigehito Mori \\ morij@rm.rcn.ne.jp \\ Yukihiro Kimura \\ kimyuki@u-fukui.a.jp \\ Takahiro Tokunaga \\ t-toku3@nifty.com \\ Yukinori Kato \\ ykato@u-fukui.ac.jp
}

Tetsuji Takabayashi

tetsuji@u-fukui.ac.jp

Norihiko Narita

norihiko@u-fukui.ac.jp

Shigeharu Fujieda

sfujieda@u-fukui.ac.jp

1 Division of Otorhinolaryngology, Shinseikai Toyama Hospital, 89-10, Shimowaka, Imizu City, Toyama 939-0243, Japan

2 Division of Otorhinolaryngology Head and Neck Surgery, Department of Sensory and Locomotor Medicine, Faculty of Medical Sciences, University of Fukui, Fukui 910-1193, Japan

3 MORI Otorhinolaryngology and Allergy Clinic, Fukui 914-0124, Japan 


\section{Background}

Allergic rhinitis (AR) is increasingly common in Japan; in a 2010 study involving 1540 participants, we estimated that the prevalence of this disease is $44 \%$ [1]. Furthermore, the estimated annual economic loss due to seasonal AR caused by Japanese cedar pollen is over $\$ 3$ billion. For this reason, AR is regarded as a major public health problem in Japan [2, 3]. In addition, the number of patients with AR is increasing worldwide $[4,5]$. Medical treatments such as antihistamines and topical corticosteroids are prescribed to patients with AR as a first step strategy. However, AR and vasomotor rhinitis (VR) are intractable to medication therapy in some patients, and surgical intervention should be considered as a treatment option.

In 1961, Golding-Wood introduced trans-maxillary sinus vidian neurectomy to treat intractable VR [6]. Since then, several modified approaches to this procedure have been reported, such as transpalatal [7], trans-septal [8], and transsphenoidal variations [9]. Moreover, the indications for the operation have been expanded to include perennial AR. However, the original method was abandoned in the beginning of the 1980s because it is difficult and can cause irreversible complications [10].

Recent advances in endoscopic technology have afforded surgeons a clear view of the paranasal sinus and allowed safe access to the sphenopalatine foramen, which is a landmark of the posterior nasal nerve. The posterior nasal nerve, which is a peripheral branch of the vidian nerve, carries secretory fibers and sensory nerve fibers of the parasympathetic and sympathetic systems to the nasal respiratory mucosa. Posterior nasal neurectomy (PNN) aims to disrupt autonomic supply, with a reduction in excessive nasal secretions. Endoscopic transnasal PNN circumvented the complications of vidian neurectomy, which include dry eyes, visual loss, and palatal numbness.

Alternatively, submucous inferior turbinectomy (ST) can also be used to treat patients with refractory AR. It is one of several turbinoplasty techniques that have been performed; others include partial or total turbinate resection, cryosurgery, and laser ablation [11-15]. ST has several advantages. For instance, removal of the inferior turbinate bone reduces both inferior turbinate volume and infiltration of various inflammatory or allergy-related cells [12]. In addition, the procedure leaves the overlying mucosa intact, preserving epithelial and ciliary function.

In the present study, we combined endoscopic ST with PNN to treat patients with intractable AR unresponsive to medical therapy. The study assessed the long-term effects and complications of this combined surgical intervention.

\section{Methods}

\section{Subjects}

The constitution of our subjects was shown in Table 1. In total, we enrolled 127 patients who had undergone endoscopic ST combined with PNN in our hospital between July 2006 and June 2013. We mailed these patients card questionnaires, and 62 valid card questionnaires were obtained from 127 subjects. Of these questionnaires, we excluded the 5 patients with VR and 26 patients who had undergone concomitant endoscopic sinus surgery (ESS), because they had both AR and sinusitis. Thus, 31 valid card questionnaires were ultimately obtained from AR patients who had undergone combined ST and PNN only. We assessed the cases which were followed up more than 1 year. We mailed same questionnaires to 147 patients who had not undergone ST combined PNN but had undergone ESS only as a control group. We obtained 74 valid answers from the control group to investigate complication, which is numbness of the palate, cheeks, or teeth.

Then, we investigated 31 patients' symptoms. AR to seasonal or perennial allergens (cedar pollen or house dust mite) was confirmed using a radioallergosorbent test (RAST). Only patients with a RAST score of class 2 or greater were included in the AR category. The patients with VR had a history of intractable rhinitis and showed a negative RAST for seasonal and perennial allergens common in Japan.

To avoid the pollen season (March and April), we mailed the questionnaires in the month of June.

\section{Surgical Procedure}

The ST procedure has previously described in detail [12]. Briefly, the patients were placed under local anesthesia by injection with $0.5 \%$ lidocaine and 1:10,000 adrenaline. An incision into the nasal mucosa was then made along with the edge of the aperture piriformis, and the turbinate bone was isolated and removed.

The PNN was also carried out according to previous reports $[16,17]$. In brief, patients were placed in the supine position under general anesthesia. Surgical gauze soaked in

Table 1 Constitution of the subjects. Assessed subjects were indicated by italics

\begin{tabular}{lllll}
\hline Combined ST and PNN & + & & - & \\
& 127 & & 147 & \\
Card questionnaire & + & - & + & - \\
& 62 & 65 & 74 & 72 \\
VR & 5 & & & \\
Concomitant ESS & 26 & & & \\
AR & 31 & & & \\
\end{tabular}


Table 2 Results of the QOL investigation using the card questionnaire. $Q O L$, quality of life

\section{AR}

Present nasal allergic symptoms in comparison with those at pre-operative state

\begin{tabular}{ll}
\hline Excellent & $7(22.6 \%)$ \\
Good & $17(54.8 \%)$ \\
Not changed & $7(22.6 \%)$ \\
Worth & 0 \\
\hline
\end{tabular}

epinephrine was used to shrink the middle turbinate, inferior turbinate, and open middle nasal meatus, for improving visualization. Local anesthesia was administered at the posterior end of middle turbinate using $0.5 \%$ lidocaine and 1:10,000 adrenaline. An incision was then made in the posterior end of the membranous portion of the maxillary sinus. A mucoperiosteum flap was isolated from the lateral bony wall, and the sphenopalatine foramen was identified at the posterior end of the middle turbinate. Next, the sphenopalatine artery and nerve bundles running parallel were identified in the sphenopalatine foramen. In some cases, we could identify posterior nasal nerve without a second incision in the posterior end of the membranous portion of the maxillary sinus, because extensive mucoperiosteum flap isolation after ST enabled to find the nerve. The identified posterior nerve was coagulated and resected with the sphenopalatine artery using an ultrasonically activated scalpel. The procedure was performed bilaterally, and the nasal packing materials were removed on the second day after the surgery.

\section{Analysis of Symptom and Complications}

The mean daily frequency of sneezing and rhinorrhea, as well as a subjective grade for nasal obstruction, was evaluated using a numerical scoring system. For both sneezing and rhinorrhea, the grades were 0 (none), 1 (1-4 times per day), 2 (5-9 times per day), and 3 (more than 10 times per day). For nasal obstruction, grades were 0 (none), 1 (mild), 2 (moderate), and 3 (severe). In addition, we investigated the patients' quality of life (QOL), as well as their nasal symptoms scores, using the mail questionnaire. Two questions with multiplechoice answers were asked as follows: (1) how do you feel about your allergic symptoms now in comparison with those before the operation?-(a) Excellent, (b) good, (c) not changed, (d) worse; (2) have you required postoperative treatment for your nose? - (a) No, (b) need over the counter medicine occasionally, (c) sometimes consult an otolaryngology clinic (once or twice every few months), (d) receive treatment from an otolaryngology clinic every month.

Numbness of the palate, cheeks, or teeth was assessed in the card questionnaire using a numerical scoring system as follows: 0 (none), 1 (transient numbness), 2 (mild persistent

numbness), 3 (severe persistent numbness). We compared the answers from whom the patient had undergone PNN or not among the 136 returned card questionnaires (PNN $(+), n=62$; PNN (-), $n=74)$. In the 127 patients who had undergone PNN, postoperative bleeding was investigated using the medical records.

The present study was performed in accordance with the Declaration of Helsinki. All procedures were approved by the ethical review board of the Shinseikai Toyama Hospital.

\section{Statistical Analysis}

All data analyses were performed by Wilcoxon's test and Mann-Whitney test using GraphPad Software (California, USA). $P$ values $<0.05$ were considered statistically significant.

\section{Results}

\section{Results of Quality of Life Investigation}

First, we assessed the quality of life of patients whom more than 1 year had passed since the operation (Table 2). In the 31 valid questionnaires of AR patients who had undergone combined ST and PNN, seven patients (22.6\%) answered "excellent," seventeen (54.8\%) answered "good," seven (22.6\%) answered "not changed," and none (0\%) answered "worse" to the question "How do you feel about your nasal symptoms now compared to before the operation?" Regarding postoperative treatment for AR, although five patients had been receiving medical treatment from an otolaryngology clinic every month before the operation, none received any such treatment after the operation (Table 3 ).

\section{Subjective Symptom Scores}

One year after the operation $(n=31)$, symptom scores were significantly lower than the re-operative values for nasal sneezing $(1.03 \pm 0.20$ vs. $0.56 \pm 0.13, P<0.01)$, rhinorrhea (1.92 \pm 0.19 vs. $1.14 \pm 0.14, P<0.01)$, and nasal obstruction $(2.42 \pm 0.15$ vs. $1.22 \pm 0.14, P<0.01)$ (Fig. 1$)$. Three years after the operation, among 19 patients whom more than 3 years had passed since the operation, the scores were slightly higher than those 1 year after the operation. Nonetheless, the mean scores were still lower than the pre-operative levels. Regarding individual symptoms, the scores 1 and 3 years after the operation were significantly lower than the pre-operative values, although they did increase gradually after surgery. We found no significant reduction in sneezing and rhinorrhea 3 years after the operation, although there was a significant improvement in nasal obstruction after postoperative 1 year $(P<0.01)$, more than 3 years $(P<0.01)$. However, there was 
Table 3 Results of the investigation into the requirements for nasal allergy treatment. $Q O L$, quality of life; $E N T$, ear, nose, and throat

\begin{tabular}{lcc}
\hline & Preoperation & Postoperation \\
\hline Nothing & $7(22.6 \%)$ & $17(54.8 \%)$ \\
Need over the counter medicine occasionary & $8(25.8 \%)$ & $5(16.1 \%)$ \\
Sometimes consult an ENT clinic & $11(35.5 \%)$ & $9(29.0 \%)$ \\
Receiving treatment from an ENT clinic every month & $5(16.1 \%)$ & 0 \\
\hline
\end{tabular}

no significant improvement of the group which had passed more than 6 years after an operation in all symptoms.

\section{Complication}

The rates of postoperative bleeding were investigated using medical records in 127 patients who had undergone PNN during the study period. Five patients $(3.9 \%)$ had been treated for postoperative bleeding from the sphenopalatine artery (Table 4), all of whom had experienced the bleeding more than 7 days after surgery. Four of these patients were admitted to the hospital, and three required repeat surgery under general anesthesia. Furthermore, we found that youth was a risk factor for postoperative bleeding.

Next, numbness of the palate, cheeks, or teeth was assessed among the 136 returned card questionnaires (Fig. 2). Specifically, the patients that had undergone PNN, PNN (+) $(n=62)$ and PNN $(-)(n=74)$, were compared with those that had undergone ESS only. Three patients $(4.8 \%)$ in the PNN (+) group complained of severe persistent numbness, while two patients $(2.7 \%)$ in the ESS only; PNN (-) group did so. There was no significant difference between the two groups in this regard. The prevalence of postoperative persistent numbness of the palate or teeth was $11.2 \%$ in the PNN (+) group and $10.8 \%$ in the PNN (-) group; this difference was also not significant. These results suggest that there is no relationship between PNN and numbness of the palate, cheeks, or teeth.

\section{Discussion}

In this study, there was a significant improvement in all nasal symptoms after combined ST and PNN on AR. The card

Table 4 Cases of postoperative bleeding. POD, post-operation day; $S P A$, sphenopalatine artery

\begin{tabular}{llrll}
\hline $\begin{array}{l}\text { Case } \\
\text { age }\end{array}$ & $\begin{array}{l}\text { Sex/ } \\
\text { POD }\end{array}$ & Artery & Treatment \\
\hline 1 & $\mathrm{M} / 22$ & 10 & Rt SPA & Admission, operation \\
2 & $\mathrm{M} / 54$ & 7 & Lt SPA & Packing \\
3 & $\mathrm{M} / 16$ & 18 & Rt SPA & Admission, packing \\
4 & $\mathrm{M} / 16$ & 8 & Bil SPA & Admission, operation \\
5 & $\mathrm{M} / 19$ & 10 & Rt SPA & Admission, operation \\
\hline
\end{tabular}

questionnaire showed that the combined ST and PNN had an effect in $77.4 \%$ of the AR patient; $70.9 \%$ of the AR patients did not visit otorhinolaryngology clinics postoperatively (Tables 2 and 3). Despite the difference was not significant in statistics, the answer from VR patients was also good (data not shown). One year after surgery, the mean scores for each nasal symptom were statistically lower than their preoperative levels. However, after more than 6 years, there was no significant improvement in all symptoms (Fig. 1). Although the score of nasal obstruction remained significantly improved after more than 6 years, the sneezing and rhinorrhea increased over time. It follows that regeneration of resected nerve may affect sneezing and rhinorrhea score. However, it is considered that ST, which can reduce inferior turbinate volume, have a long-lasting effect on nasal obstruction. Nasal obstruction is regarded as the most important symptoms among these three symptoms from our result (Fig. 1). Moreover, the previous study in animals found that PNN effectively suppresses nasal secretion, although it does not affect mucosal thickening or AR hypersensitivity [18]. Therefore, we considered that combined ST and PNN, not PNN alone, are the best intervention to provide long-lasting alleviation of nasal symptoms, especially nasal obstruction and rhinorrhea. A prospective randomized study in 3 groups (ST, PNN, and combined ST and PNN) is required to reveal the further effect of combined ST and PNN.

Kobayashi et al. emphasized that selective resection of peripheral branches of the posterior nasal nerve with submucous turbinoplasty can reduce allergic symptoms as effectively as total resection of the posterior nasal nerve [18]. However, they also reported that the patients who had undergone total resection of the posterior nasal nerve had higher improvements in their nasal symptom scores, although the difference was not significant. By way of explanation, the authors speculated that total resection of the posterior nasal nerve results in denervation of the middle turbinate and nasal septum. In fact, resection of the peripheral branches of the posterior nasal nerve is safer than resection of the posterior nasal nerve trunk at the sphenopalatine foramen. However, we should consider the evidence carefully in terms of long-lasting effect. Despite the symptomatic improvements and low complication rate associated with PNN, some patients did not have a complete response after PNN. This may be because of gradual postoperative reinnervation, or to the presence of persistent accessory secretomotor fibers to the posterolateral mucosa that do not 
Fig. 1 Chronological changes in nasal symptom scores from the card questionnaire (pre-operative, postoperative 1 year, 3 years, and 6 years). $(* * P<0.01)$

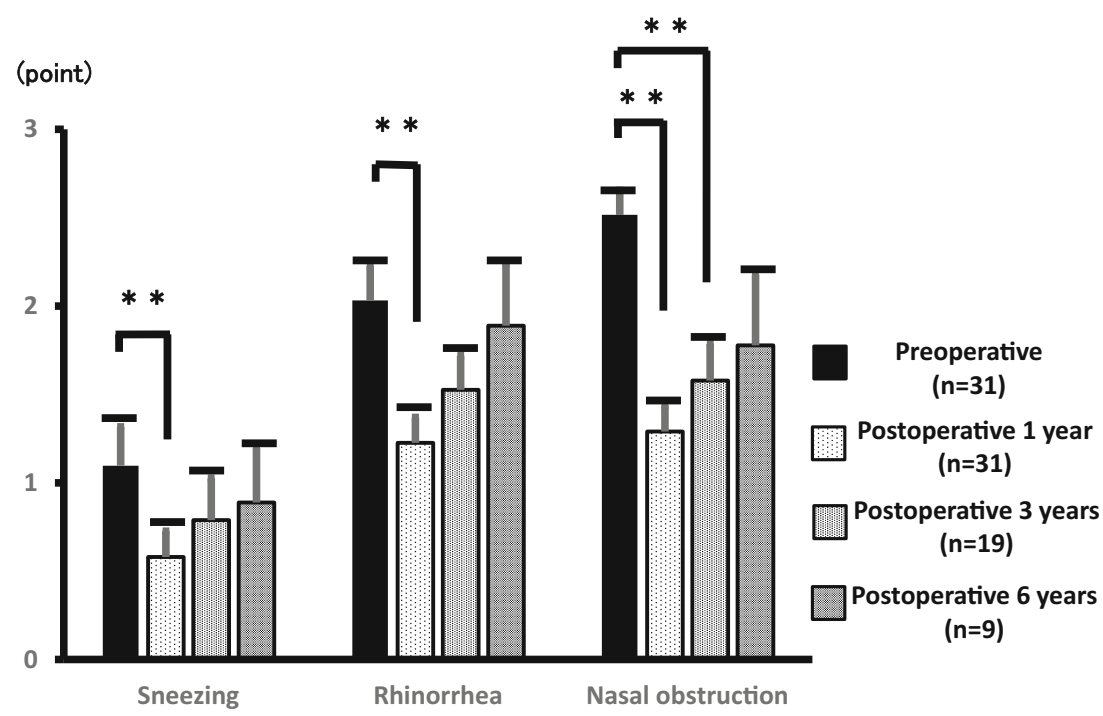

transverse the sphenopalatine foramen [19]. Robinson and Wormald suggested that surgeons must remove a segment of the nerve and cauterize the nerve stump to prevent regeneration [20]. Others have placed bone wax or gel foam on the resected nerve stump to prevent regeneration [21]. The same authors reported the effects of vidian neurectomy last for 7 years or more. In our hospital, before this study was conducted, we had placed nothing on the nerve stump to prevent regeneration. Therefore, it may be that the symptom scores increased over time due to nerve regeneration (Fig. 1). Albu et al. stated that the addition of PNN appears to offer no additional benefit in the subjective and objective outcome related to inferior turbinoplasty [22]. However, they just cauterized the bundle and did not elaborate on any schemes to prevent nerve regeneration. We should consider a new strategy which prevents postoperative reinnervation in order to get better results.

According to the previous report, postoperative bleeding is frequently encountered in the cases conducted PNN compared to the cases conducted inferior turbinoplasty only [22]. However, the posterior inferior nasal nerve was consistently found posterior to the sphenopalatine artery in another previous study [23]. Therefore, in most cases, the sphenopalatine artery is difficult to preserve and must be resected along with the posterior nasal nerve to ensure that the latter is cut. In actuality, we resected posterior nasal nerve with a sphenopalatine artery in all 127 subjects. The entrance was too narrow to insert an ultrasonically activated scalpel using the trans-turbinate approach. In several cases, to improve visualization, we were forced to add an additional incision at the posterior end of the middle nasal meatus. This additional incision was wide enough to insert the ultrasonically activated scalpel and closer to sphenopalatine foramen; therefore making it easier to approach the sphenopalatine foramen, however, increased the rate of postoperative bleeding. Therefore, to avoid postoperative bleeding, it would be better to use the trans-turbinate approach after ST, rather than making an additional incision. In addition, to prevent postoperative bleeding, the elevated mucoperiosteum flap should be replaced to cover the vessel stump. For the same reason, tension should not be transferred to the vessel when the bundle is resected using an ultrasonically activated scalpel, and surgeons should allow sufficient coagulation before resecting the fascicle.

In the present study, several patients in the ESS and ST/ PNN groups complained of numbness in the palate, cheek, or teeth (Fig. 2). In another study, 22.2\% of patients who had
Fig. 2 Comparison of postoperative numbness of the palate or teeth

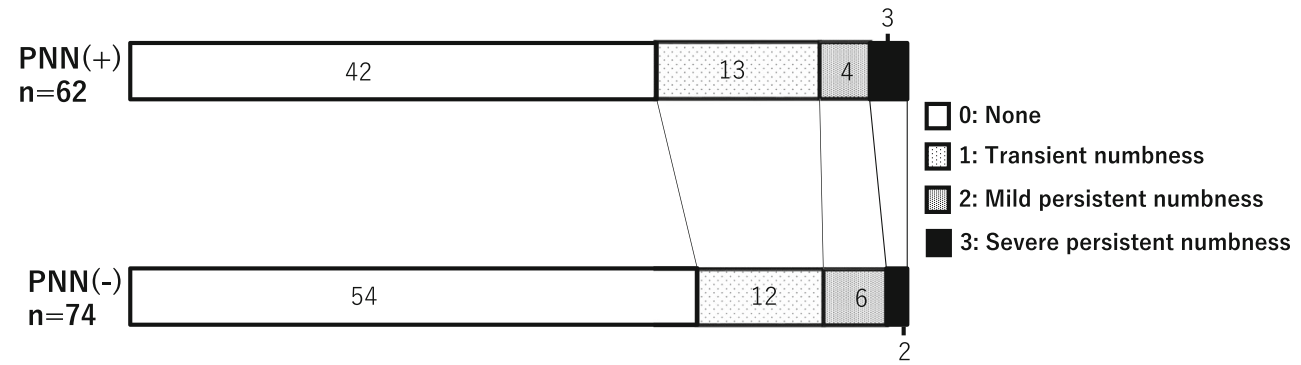

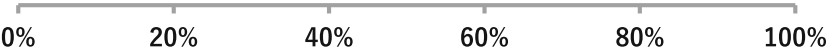


undergone vidian neurectomy complained of mild transient numbness in palate and lip [24]. It seems that the procedure damaged the superior alveolar nerve, which is a peripheral branch of the maxillary nerve and that septoplasty affects the superior alveolar nerve. Alternatively, these patients might have an anomalous trigeminal nerve. None of the patients who had undergone PNN in the present study complained of dry eye, which has been reported after vidian neurectomy.

\section{Conclusions}

In conclusion, we found that endoscopic ST combined with PNN is an effective surgery to treat intractable AR. However, further prospective well-designed study is needed to validate our results. In addition, the indications for surgery to treat intractable rhinitis should be considered in terms of the balance between long-term efficacy and complications. It remains controversial whether the sphenopalatine artery should be preserved to prevent postoperative bleeding or resected to avoid nerve regeneration. In any case, the most beneficial treatment should be considered based on the basis of evidence from further research, as well as the demands of each patient.

Acknowledgment The authors wish to thank Mr. S Takubo for his assistance. This work was supported by JSPS KAKENHI (Grants-in-Aid for Scientific Research from the Ministry of Education, Culture, Sports, Science and Technology of Japan; Grant Number 16K20237, JP18K16881).

Authors' Contributions $\mathrm{KO}$ and YM designed the study; YK, TTO, and YK collected and analyzed the data; TTA and NN wrote the manuscript; SM and SF revised and critically appraised it. KO drafted the first version of the manuscript. All authors contributed to conception and design, analysis, and interpretation of data, revising it critically for important intellectual content. All authors read and approved the final manuscript.

Funding The authors declare that this study was carried out with institutional resources only.

Data Availability All data are available upon request.

\section{Compliance with Ethical Standards}

Ethics Approval and Consent to Participate All procedures performed in studies involving human participants were in accordance with the ethical standards of the institutional and/or national research committee and with the 1964 Helsinki Declaration and its later amendments or comparable ethical standards. Written informed consent was obtained from all individual participants included in the study. All procedures were approved by the ethical review board of the Shinseikai Toyama Hospital.

Consent for Publication The manuscript has been approved by all the authors. All authors concur with the submission.

Conflict Interest The authors declare that they have no conflict interests.
Open Access This article is distributed under the terms of the Creative Commons Attribution 4.0 International License (http://creativecommons. org/licenses/by/4.0/), which permits unrestricted use, distribution, and reproduction in any medium, provided you give appropriate credit to the original author(s) and the source, provide a link to the Creative Commons license, and indicate if changes were made. The Creative Commons Public Domain Dedication waiver (http://creativecommons. org/publicdomain/zero/1.0/) applies to the data made available in this article unless otherwise stated.

Abbreviations AR, allergic rhinitis; VR, vasomotor rhinitis; PNN, posterior nasal neurectomy; ST, submucous inferior turbinectomy; ESS, endoscopic sinus surgery; RAST, radioallergosorbent test

Open Access This article is distributed under the terms of the Creative Commons Attribution 4.0 International License (http:// creativecommons.org/licenses/by/4.0/), which permits unrestricted use, distribution, and reproduction in any medium, provided you give appropriate credit to the original author(s) and the source, provide a link to the Creative Commons license, and indicate if changes were made.

\section{References}

1. Sakashita M, Hirota T, Harada M, Nakamichi R, Tsunoda T, Osawa $\mathrm{Y}$, et al. Prevalence of allergic rhinitis and sensitization to common aeroallergens in a Japanese population. Int Arch Allergy Immunol. 2010;151(3):255-61.

2. Yamada T, Saito H, Fujieda S. Present state of Japanese cedar pollinosis: the national affliction. J Allergy Clin Immunol. 2014;133(3):632-9 e635.

3. Okubo K, Kurono Y, Fujieda S, Ogino S, Uchio E, Odajima H, et al. Japanese guideline for allergic rhinitis 2014. Allergol Int. 2014;63(3):357-75.

4. Bousquet J, Khaltaev N, Cruz AA, Denburg J, Fokkens WJ, Togias A, et al. Allergic rhinitis and its impact on asthma (ARIA) 2008 update (in collaboration with the World Health Organization, GA(2)LEN and AllerGen). Allergy. 2008;63(Suppl 86):8-160.

5. Singh K, Axelrod S, Bielory L. The epidemiology of ocular and nasal allergy in the United States, 1988-1994. J Allergy Clin Immunol. 2010;126(4):778-83 e776.

6. Golding-Wood PH. Observations on petrosal and vidian neurectomy in chronic vasomotor rhinitis. J Laryngol Otol. 1961;75:232-47.

7. Chandra R. Transpalatal approach for vidian neurectomy. Arch Otolaryngol. 1969;89(3):542-5.

8. Minnis NL, Morrison AW. Trans-septal approach for vidian neurectomy. J Laryngol Otol. 1971;85(3):255-60.

9. Lee JC, Kao CH, Hsu CH, Lin YS. Endoscopic transsphenoidal vidian neurectomy. Eur Arch Otorhinolaryngol. 2011;268(6):851-6.

10. Krant JN, Wildervanck de Blécourt P, Dieges PH, de Heer LJ. Long-term results of vidian neurectomy. Rhinology. 1979;17(4): 231-5.

11. Passàli D, Lauriello M, Anselmi M, Bellussi L. Treatment of hypertrophy of the inferior turbinate: long-term results in 382 patients randomly assigned to therapy. Ann Otol Rhinol Laryngol. 1999;108(6):569-75.

12. Mori S, Fujieda S, Igarashi M, Fan GK, Saito H. Submucous turbinectomy decreases not only nasal stiffness but also sneezing and rhinorrhea in patients with perennial allergic rhinitis. Clin Exp Allergy. 1999;29(11):1542-8.

13. Martinez SA, Nissen AJ, Stock CR, Tesmer T. Nasal turbinate resection for relief of nasal obstruction. Laryngoscope. 1983;93(7):871-5. 
14. Moore JR, Bicknell PG. A comparison of cryosurgery and submucous diathermy in vasomotor rhinitis. J Laryngol Otol. 1980;94(12):1411-3.

15. Lippert BM, Werner JA. Comparison of carbon dioxide and neodymium: yttrium-aluminum-garnet lasers in surgery of the inferior turbinate. Ann Otol Rhinol Laryngol. 1997;106(12):1036-42.

16. Ogawa T, Takeno S, Ishino T, Hirakawa K. Submucous turbinectomy combined with posterior nasal neurectomy in the management of severe allergic rhinitis: clinical outcomes and local cytokine changes. Auris Nasus Larynx. 2007;34(3):319-26.

17. Ikeda K, Yokoi H, Saito T, Kawano K, Yao T, Furukawa M, et al. Prevention of ultrasonic coagulator-mediated mucoperiosteal flap injury and defects by using a clip manipulation in the resection of the posterior nasal nerve. Rhinology. 2009;47(1):45-7.

18. Kobayashi T, Hyodo M, Nakamura K, Komobuchi H, Honda N. Resection of peripheral branches of the posterior nasal nerve compared to conventional posterior neurectomy in severe allergic rhinitis. Auris Nasus Larynx. 2012;39(6):593-6.

19. Nishijima H, Kondo K, Toma-Hirano M, Iwasaki S, Kikuta S, Fujimoto C, et al. Denervation of nasal mucosa induced by posterior nasal neurectomy suppresses nasal secretion, not hypersensitivity, in an allergic rhinitis rat model. Lab Investig. 2016;96(9):981-93.

20. Robinson SR, Wormald PJ. Endoscopic vidian neurectomy. Am J Rhinol. 2006;20(2):197-202.

21. Jang TY, Kim YH, Shin SH. Long-term effectiveness and safety of endoscopic vidian neurectomy for the treatment of intractable rhinitis. Clin Exp Otorhinolaryngol. 2010;3(4):212-6.

22. Albu S, Trombitas V, Nagy A. Endoscopic microdebrider-assisted inferior turbinoplasty with and without posterior nasal neurectomy. Auris Nasus Larynx. 2014;41(3):273-7.

23. Eren E, Zeybek G, Ecevit C, Arslanoğlu S, Ergur I, Kiray A. A new method of identifying the posterior inferior nasal nerve: implications for posterior nasal neurectomy. J Craniofac Surg. 2015;26(3): 930-2.

24. Ma Y, Tan G, Zhao Z, Li W, Huang L, Liu G. Therapeutic effectiveness of endoscopic vidian neurectomy for the treatment of vasomotor rhinitis. Acta Otolaryngol. 2014;134(3):260-7.

Publisher's Note Springer Nature remains neutral with regard to jurisdictional claims in published maps and institutional affiliations. 